\title{
Microevolution events in Allioideae (Amaryllidaceae) plastomes
}

Scobeyeva V.A. ${ }^{1,2}$, Artyushin I.V. ${ }^{1}$, Krinitsina A.A. ${ }^{1}$, Nikitin P.A. ${ }^{1 *}$, Antipin M.I. ${ }^{1}$, Kuptsov S.V. ${ }^{1}$, Belenikin M.S. ${ }^{2}$, Omelchenko D.O. ${ }^{3}$, Logacheva M.D. ${ }^{4}$, Konorov E.A. ${ }^{5}$, Samoilov A.E. ${ }^{6}$, Speranskaya A.S. ${ }^{1,6}$

${ }^{1}$ Lomonosov Moscow State University, Moscow, Russia

${ }^{2}$ Moscow Institute of Physics and Technology, Moscow, Russia

${ }^{3}$ Institute for Information Transmission Problems (RAS), Moscow, Russia

${ }^{4}$ Center of Life Sciences, Skolkovo Institute of Science and Technology, Moscow, Russia

${ }^{5}$ Vavilov Institute of General Genetics, Russian Academy of Sciences, Moscow, Russia

${ }^{6}$ Central Research Institute of Epidemiology (CRIE), Moscow, Russia

*email:niki4pingvi@mail.ru

Amaryllidaceae is a large family with more than 1600 species, belonging to 75 genera. The largest genus - Allium - is vast, comprising about a thousand species. Allium species (as well as other members of the Amaryllidaceae) are widespread and diversified, they are adapted to a wide range of habitats from shady forests to open habitats like meadows, steppes and deserts. The genes present in chloroplast genomes (plastomes) play fundamental roles for the photosynthetic plants [1]. Plastome traits could thus be associated with geophysical abiotic characteristics of habitats. Most chloroplast genes are highly conserved and are used as phylogenetic markers for many families of vascular plants. Nevertheless some studies revealed signatures of positive selection in chloroplast genes of many plant families including Amaryllidaceae. In this work we provide analysis of Allioideae subfamily plastid genomes microevolutionary events. First of all, we prepared primary cpDNA alignment of 38 species of Allium genera (including all the three evolutionary lines [2]) and 11 other species of Amaryllidaceae family as an outgroup, both provided by our group and NCBI GenBank. Then we constructed a maximum likelihood phylogenetic tree with IQ-Tree2. With this data we got some information of relative tree length in alignment blocks and $\mathrm{dN} / \mathrm{dS}$ rate for evaluating the balance between neutral mutations and positive/negative natural selection. Sites under selection were found with aBSREL (adaptive Branch-Site Random Effects Likelihood); FUBAR (Fast Unconstrained Bayesian AppRoximation) и MEME (Mixed Effects Model of Evolution) as well. We found that infA, ccsA, rps 2 and rps 16 genes have lost their functionality multiple times in different species (regularly evolutionary events), while the pseudogenization of other genes was stochastic events. We found that the "normal" or "pseudo" state of rps2, infA, rps 16 and $\operatorname{css} A$ genes correlates well with the evolutionary line of genus the species belongs to. Independent methods revealed some genes being under positive selection, such as housekeeping genes (accD, ccsA, rpll6, rpl20, rps16, rpoA, rрoB, matK). The most interestingly, the part of photosynthesisinvolved genes were also found under positive selection (various $n d h$ genes, $p s b M$ and $r b c L)$ as well as genes of unknown function ( $y c f 1, y c f 2)$. Taking into account known mechanisms of coping with excessive light by cyclic electron transport, we can hypothesize that adaptive evolution in genes, coding subunits of NADH-plastoquinone oxidoreductase could be driven by abiotic factors like temperature, light intensity or UV radiation [3].

References

1 Daniell H., Lin C.-S., Yu M., Chang W.-J. Gen. Biol. 2016;17:134.

2 Friesen N., Fritsch R.M., Blattner F.R. Aliso: Journal Systematic Evolutionary Botany. 2006;22:372-395.

3 Martín M., Sabater B. Plant Physiol. Biochemistry. 2010;48:636-645. 